\title{
miR-125b-5p is A Promising Novel Plasma Biomarker for Alveolar Echinococcosis in Patients from the Southern Province of Qinghai
}

Deping Cao ( $\sim$ qhmccdp@163.com )

Guilin Medical University https://orcid.org/0000-0002-1973-8879

\section{Bofan Jiang}

Qinghai university medical college

Yaogang Zhang

Affliated hospital of Qinghai unniversity

Mingquan Pang

affiliated hospital of Qinghai university

Research article

Keywords: alveolar echinococcosis, diagnostic biomarker, hsa-miR-125b-5p, plasma, hepatic tissue samples

Posted Date: September 18th, 2020

DOl: https://doi.org/10.21203/rs.3.rs-68399/v1

License: (c) (i) This work is licensed under a Creative Commons Attribution 4.0 International License. Read Full License

Version of Record: A version of this preprint was published on March 7th, 2021. See the published version at https://doi.org/10.1186/s12879-021-05940-z. 


\section{Abstract}

Background: Alveolar echinococcosis is an infectious zoonotic disease caused by Echinococcus multilocularis that is endemic to the vast pastoral areas of southern Qinghai province of China. Alveolar echinococcosis affects the human liver, and its clinical manifestations are similar to those of liver tumors. It is also called "worm cancer," as it induces irreversible liver fibrosis and brain metastases. Alveolar echinococcosis is a serious threat to human health and is a burden to patients and economies. When detected and treated early, patients with alveolar echinococcosis have a strong chance of recovery. Therefore, it is imperative to identify early diagnostic biomarkers of the disease. MicroRNAs (miRNAs) are short, single-stranded, non-coding RNA molecules that play key roles in a wide range of biological processes, and have been recently implicated in tumorigenesis. However, little is known about the role of miRNAs in alveolar echinococcosis. We have previously reported differentially expressed miRNAs in the plasma of patients with alveolar echinococcosis and healthy individuals, using microarray assay chips.

Methods: We screened 10 differentially expressed miRNAs based on their fold change and the available literature. Additionally, quantitative polymerase chain reaction was used to verify their expression in the plasma of alveolar echinococcosis patients. We found that hsa-miR-125b-5p was upregulated in the plasma and the hepatic tissue samples of alveolar echinococcosis patients; therefore, its role as a diagnostic biomarker for alveolar echinococcosis was investigated. Accordingly, hsa-miR-125b-5p was upregulated in plasma and hepatic tissue samples obtained from alveolar echinococcosis patients. Receiver operating characteristic curves showed that hsa-miR-125b-5p was associated with an area under the curve of $99.8 \%$ and $98.9 \%$ in the plasma and hepatic tissues of alveolar echinococcosis patients, respectively. Transfection of an LV-hsa-miR-125b-5p-inhibitor reduced apoptosis of L-02 hepatocytes in vitro $(P<0.05)$. Thus, hsa-miR-125b-5p may promote liver cell proliferation and inhibit liver cell apoptosis.

Conclusions: Taken together, hsa-miR-125b-5p may be a promising diagnostic biomarker for the early non-invasive diagnosis of alveolar echinococcosis. We plan to validate the expression of hsa-miR-125b$5 p$ in the plasma of patients with cystic echinococcosis, alveolar echinococcosis, and other liver diseases to determine the potential of hsa-miR-125-5p as a differential biomarker in future studies.

\section{Highlights}

- Hsa-miR-125b-5p was upregulated in samples from alveolar echinococcosis patients.

- L-02 hepatocytes depleted of hsa-miR-125b-5p show increased cell viability.

- L-02 hepatocytes depleted of hsa-miR-125b-5p correlated with decreased apoptosis.

- Hsa-miR-125b-5p may be a promising novel biomarker for alveolar echinococcosis.

\section{Background}


Echinococcus multilocularis causes alveolar echinococcosis, one of the most lethal parasitic infections in humans ${ }^{[1]}$. Alveolar echinococcosis is a zoonotic disease that can be found globally, particularly where animal husbandry is practiced. It is particularly endemic to countries with developed animal husbandry, such as the northwest and Tibetan plateau of China, especially the Qinghai Tibetan plateau, where mixed infections of cystic echinococcosis and alveolar echinococcosis ${ }^{[2]}$. Alveolar echinococcosis primarily develops in the liver of intermediate hosts. Humans, primates, and other accidental hosts become infected upon ingestion of parasite eggs present in the feces of canine hosts (e.g., dogs and foxes), exposure to egg-contaminated environments, or consumption of contaminated food or drinking water ${ }^{[3,4}$, 5]. When left untreated, alveolar echinococcosis is associated with 5- and 10-years mortality rates of $52 \%$ and $96 \%$, respectively ${ }^{[6]}$. Owing to the tumor-like growth of metacestodes (also called "worm carcinoma") in the liver and challenging early diagnosis and treatment ${ }^{[7]}$, alveolar echinococcosis is a significant public health concern that poses an economic burden for patients and their families in the southern region of the Qinghai province ${ }^{[8]}$. The current protocol for diagnosing alveolar echinococcosis predominantly relies on medical history, imaging, and immunological assays. However, none of these methods is accurate and there are no specific and sensitive molecular markers for the early diagnosis of alveolar echinococcosis ${ }^{[9]}$.

MicroRNAs (miRNAs) are short, endogenous, non-coding RNAs, that post-transcriptionally regulate gene expression by binding to the $3^{\prime}$-untranslated regions of target mRNAs ${ }^{[10]}$. Parasite-resident miRNAs are essential for cellular invasion, development, and ability of the host to respond to environmental and developmental signals ${ }^{[11]}$. miR-125b-5p upregulation is associated with the severity of liver damage, and high serum miR-125b-5p levels may serve as a predictor for poor outcomes in hepatitis B virus associated acute-on-chronic liver failure cases ${ }^{[12]}$. Consistent with other reports, Wang ${ }^{[13]}$ et al. have shown that circulating exosomes are promising candidates for diagnostic and prognostic biomarkers of human cancers. Furthermore, miR-125-3p was shown to enhance the diagnostic potential of carcinoembryonic antigen for early stage colon cancer ${ }^{[13]}$.

\section{Methods}

\subsection{Plasma from alveolar echinococcosis patients and healthy individuals}

We collected plasma from 44 alveolar echinococcosis patients and 44 healthy individuals (22 males and 22 females) from the echinococcosis sample bank at the Qinghai University Affiliated Hospital. This study was approved by the ethics review broad of the Hospital Affiliated of Qinghai University (approval number: P-SL-2019054). All patients were diagnosed based on their clinical symptoms and imaging data. Whole blood samples were collected in tubes, allowed to clot at $4{ }^{\circ} \mathrm{C}$ for $2 \mathrm{~h}$, and centrifuged at $12,000 \times \mathrm{g}$ for $10 \mathrm{~min}$ at $4{ }^{\circ} \mathrm{C}$. Plasma was separated from the whole blood samples, placed into fresh tubes, and centrifuged at $12,000 \times \mathrm{g}$ for $10 \mathrm{~min}$ at $4{ }^{\circ} \mathrm{C}$. Hemolyzed samples were excluded. This step was used to 
remove cell debris and blood platelets. Subsequently, the supernatant was transferred to fresh tubes and stored at $-80^{\circ} \mathrm{C}$ for further analysis.

\subsection{Collection of liver tissues from alveolar echinococcosis patients and healthy individuals}

Alveolar echinococcosis and normal liver tissues $(3 \times 3 \mathrm{~mm})$ were resected from the liver, washed three times in phosphate-buffered saline, and stored in liquid nitrogen until further analysis. Normal tissue samples were collected $2 \mathrm{~cm}$ away from the alveolar echinococcosis lesions.

\subsection{Cell culture and transfection}

L-02 hepatocytes were cultured in Dulbecco's modified Eagle medium supplemented with $10 \%$ fetal bovine serum and penicillin/streptomycin in a humidified atmosphere containing $5 \% \mathrm{CO}_{2}$ at $37^{\circ} \mathrm{C}$. Approximately $50 \mathrm{nM}$ of hsa-miR-125b-5p or control inhibitor, both purchased from JIKAI (Shanghai Jikai), was transfected into L-02 cells for $48 \mathrm{~h}$ using the riboFECTCP transfection kit (Guangzhou RIBO Biocompany) according to the protocol provided. All the experiments were performed using cells in the log phase.

\subsection{RNA extraction}

Total RNA was isolated from plasma and tissue samples using TRIzol according to the protocol provided. The concentration and integrity of isolated RNAs were evaluated using the Agilent Bioanalyzer 2100 (Agilent Technologies, Santa Clara, CA, USA). cDNA was synthesized using PrimeScript Reverse Transcriptase (TIANGEN Biotech, Beijing, Co. Ltd) according to the kit protocol. Primers targeting hsa-miR125b-5p (reverse transcription (RT): 5'-UCCCUGAGACCCUAACUUGUGA-3'; polymerase chain reaction (PCR) forward primer: 5'-TCCCTGAGACCCTAACTTGTGA-3' and PCR reverse primer: 5'-

GTGCAGGGTCCGAGGT-3') and U6 (RT primer: 5'-UGAGGUAGGAGGUUGUAUAGUU-3'; PCR forward primer: 5'-TGAGGTAGGAGGTTGTATAGTT-3'; and PCR reverse primer: 5'-GTGCAGGGTCCGAGGT-3') were purchased from Sangon Biotech (Shanghai) Co., Ltd.

\subsection{Quantitative PCR (qPCR)}

qPCR was performed using the LightCycler ${ }^{\circledR}$ 480囚 and Tiangen SYBR Green PCR Kit. U6 was used as the endogenous control for miRNA amplification. The reaction volume was $25 \mu \mathrm{l}$ and was set up according to the TB GreenTM Premix Ex TaqTM $\otimes$ protocol. Each sample and blank was analyzed in triplicate. The reaction conditions were as follows: pre-denaturation at $95^{\circ} \mathrm{C}$ for $30 \mathrm{~s}, 40$ cycles of $95^{\circ} \mathrm{C}$ for $5 \mathrm{~s}$, and $60^{\circ} \mathrm{C}$ for $30 \mathrm{~s}$. The melting curves were generated using the following temperatures: $95^{\circ} \mathrm{C}$ for $5 \mathrm{~s}$ and $60^{\circ} \mathrm{C}$ for $1 \mathrm{~min}$. hsa-miR-125b-5p expression was analyzed using the $\triangle \mathrm{Ct}$ method. Relative miRNA expression was calculated using the $2^{-} \triangle \Delta \mathrm{Ct}$ method and normalized to U6 levels.

\subsection{Inhibition of hsa-miR-125b-5p in treated L-02 hepatocytes}


L-02 hepatocytes were transfected with lentiviral vector (LV), LV-hsa-miR-125b-5p-inhibitor, and the negative control virus CON137. The L-O2 cells were transfected with the LV-hsa-miR-125b-5p inhibitor for 5 days. The transfected cells were subsequently incubated with the Cell Counting Kit-8 reagents for $2 \mathrm{~h}$ to determine the absorption at a wavelength of $450 \mathrm{~nm}$ in a time-dependent manner. $\mathrm{OD}_{450}$ represents the number of viable cells.

\subsection{Fluorescence-activated cell sorter analysis for apoptosis}

LV-hsa-miR-125b-5p-inhibition transfection solution and L-02 hepatocytes were cultured in a 6-well culture plate in triplicate. In order to ensure that a detectable number of cells was present, a cell density of $\geq 5 \times 10^{5} / \mathrm{ml}$ was used. The cell fusion was $85 \%$ on the fifth day after transfection. The cells were collected, placed into $5 \mathrm{~mL}$ centrifuge tubes, and centrifuged at $250 \mathrm{~g}$ for $5 \mathrm{~min}$ at $4^{\circ} \mathrm{C}$. The cells were washed with cold PBS, suspended in complete medium, and centrifuged at $250 \mathrm{~g}$ for $3 \mathrm{~min}$. Cells were suspended in $200 \mu \mathrm{L} 1 \times$ binding buffer, and $10 \mu \mathrm{L}$ Annexin V-APC was added to stain the cells. The cells were incubated at 10-15 min at room temperature in the dark and analyzed using BD cytoflow.

\subsection{Statistical analysis}

Data are presented as the mean \pm standard deviation. The independent sample $t$ test was used to determine the statistical differences between the groups using GraphPad Prism 8. $P<0.05$ was considered to indicate a statistically significant difference.

\section{Results}

\section{1. qPCR}

We used patient plasma to determine the potential of hsa-miR-125b-5p as an early diagnostic biomarker for alveolar echinococcosis. Our preliminary study using miRNA chips revealed upregulation of hsa-miR$125 b-5 p$.

\subsection{1 hsa-miR-125b-5p expression in plasma samples from alveolar echinococcosis patients}

qPCR showed that hsa-miR-125b-5p was upregulated in the plasma samples of alveolar echinococcosis patients $(P<0.001)$ (Table 1 and Fig. 1$)$. 
Table 1

The $2^{-\Delta \Delta C t}$ values for hsa-miR-125b-5p expression in plasma in patients with alveolar echinococcosis and controls.

\begin{tabular}{|llll|}
\hline Group & Sample & \multicolumn{2}{l|}{ hsa-miR-125b-5p } \\
\cline { 3 - 4 } & & $n$ & $2^{-\Delta \Delta C t}$ \\
\hline Alveolar echinococcosis patients & Plasma & 22 & $2.440268 \pm 0.753355$ \\
\hline Healthy controls & Plasma & 22 & $1.032328 \pm 0.261863$ \\
\hline
\end{tabular}

\subsection{2 hsa-miR-125b-5p expression in hepatic tissues from alveolar echinococcosis patients}

qPCR showed that hsa-miR-125b-5p was upregulated in the liver tissue samples obtained from alveolar echinococcosis patients $(P<0.001)$ (Table 2 and Fig. 2$)$.

Table 2

The hsa-miR-125b-5p level in hepatic tissues was statistically different between alveolar echinococcosis patients and the control group $(\mathrm{P}<$

$0.001)$.

\begin{tabular}{|llll|}
\hline Group & Sample & \multicolumn{2}{l|}{ hsa-miR-125b-5p } \\
\cline { 3 - 4 } & & $\boldsymbol{n}$ & $\mathbf{2}^{-\Delta \Delta \mathrm{ct}}$ \\
\hline Alveolar echinococcosis patients & Liver tissue & 8 & $1.932 \pm 0.682$ \\
\hline Healthy controls & Normal liver & 8 & $1.001 \pm 0.049$ \\
\hline
\end{tabular}

\subsection{Inhibition of hsa-miR-125b-5p in treated L-02 hepatocytes}

\subsubsection{Transfection of LV, LV-hsa-miR-125b-5p-inhibitor, and negative control viruses CON137 in normal L-O2 hepatocytes}

\subsubsection{Cell Counting Kit-8}

The proliferation of L-02 cells increased in the hsa-miR-125b-5p-inhibitor group compared with the negative virus control group. On the fifth day after treatment, the $\mathrm{OD}_{450}$ of the experimental group was $3.242 \pm 0.032$, while that of the control group was $1.738 \pm 0.008(P<0.05)$ (Fig. 3 and Fig. 4$)$.

\subsubsection{Cellular apoptosis}


The apoptosis (\%) of the experimental group was $4.84 \pm 0.09$, while that of the control group was $1.91 \pm$ $0.18(P<0.05)$. Apoptosis in the control and LV-hsa-miR-125b-5p inhibitor-transfected L-02 cells after 5 days was compared. Apoptosis was decreased in cells depleted of hsa-miR-125b-5p $(P<0.05)$. Thus, hsamiR-125b-5p may promote liver cell proliferation and inhibit apoptosis (Fig. 5).

The expression of miR-125b-5p was increased in the plasma and liver lesion tissues of patients with alveolar echinococcosis. Transfection of the LV-hsa-miR-125b-5p-inhibitor reduced the apoptosis of L-O2 hepatocytes in vitro. It is inferred that miR-125b-5p plays a role in alveolar echinococcosis, and may serve as a promising molecular biomarker for the diagnosis and treatment of the disease.

\section{Discussion}

miRNAs are small non-coding regulatory RNAs that play important roles in the pathogenesis of parasitic diseases $^{[14]}$. Lin $^{[15]}$ et al. (2018) found that miRNA-221/222 is upregulated in the thyroid of papillary thyroid carcinoma patients, and is crucial in invasion and lymph node metastasis, suggesting its potential as a biomarker for invasive papillary thyroid carcinoma. Mogahed ${ }^{[16]}$ et al. (2018) reported that miR-712-3p could be used as a biomarker for the early diagnosis of toxoplasmosis using qPCR and the plasma of mice acutely infected with Toxoplasma gondii.

We have previously reported differences in the expression of hsa-miR-125b-5p, hsa-let7e-5p, hsa-miR3165 , hsa-miR-4725, and hsa-miR-520e in alveolar echinococcosis plasma samples using microarray data ${ }^{[17,18]}$. In this study, 22 plasma samples from alveolar echinococcosis patients and 22 healthy controls were tested using qPCR. We observed the upregulation of hsa-miR-125b-5p in the plasma from alveolar echinococcosis patients. We also examined the expression of hsa-miR-125b-5p in liver samples obtained from 8 patients with alveolar echinococcosis and 8 controls. qPCR revealed the upregulation of hsa-miR-125b-5p in plasma and lesion tissues obtained from alveolar echinococcosis patients. We found that the area under the curve for hsa-miR-125b-5p was 0.997 in the blood, which was significantly higher than that of the control group, with a $95 \%$ confidence interval of $0.9907-1.000$ and $P<0.0001$. The area under the curve for hsa-miR-125b-5p was 0.937 in the alveolar echinococcosis liver tissues, with a 95\% confidence interval of $0.8701-1.000$ and $P<0.0001$. Thus, hsa-miR-125b-5p may serve as a useful diagnostic indicator and therapeutic target for alveolar echinococcosis.

Inhibition of hsa-miR-125b-5p in treated L-02 hepatocytes increased proliferation and decreased apoptosis. This suggested that the inhibition of hsa-miR-125b-5p may promote hepatocyte proliferation to reverse the effects of liver damage. We wish to investigate this further by determining the utility of hsamiR-125b-5p in treating mice with secondary echinococcosis.

miR-125b-5p is a member of the miR-125 family, which was first reported in 2005 by Lee ${ }^{[19]}$ et al. (2005), and regulates the proliferation of differentiated tumor cells. miR-125b-5p enhances autophagy and apoptosis in multiple myeloma cells and can be used as a novel therapeutic target and early diagnostic biomarker for multiple myeloma ${ }^{[20]}$. Thus, the role of circulating miRNAs in the early diagnosis of alveolar 
echinococcosis may improve our understanding of the pathogenesis of alveolar echinococcosis. Our findings strongly suggest that hsa-miR-125b-5p may serve as a useful diagnostic and prognostic biomarker for alveolar echinococcosis.

However, this study has certain limitations. Firstly, the findings in this study are preliminary and do not attempt to elucidate the mechanism by which hsa-miR-125b-5p is implicated in alveolar echinococcosis. Moreover, we have not addressed the association between hsa-miR-125b-5p expression and the size of the diseased tissue. Thus, the correlation between hsa-miRNA-125b-5p expression, diseased tissue size, the physiological characteristics of alveolar echinococcosis lesion(s), and recovery of patients after surgery will be investigated in future. Additionally, the potential of hsa-miRNA-125b-5p to distinguish between alveolar and cystic echinococcosis, as well as other liver diseases, requires further investigation. Our study findings require further validation using a larger cohort samples to determine whether hsa-miR$125 b-5 p$ may serve as a biomarker for the early non-invasive diagnosis of alveolar echinococcosis.

\section{Conclusions}

- In this study, we report the upregulation of hsa-miR-125b-5p in alveolar echinococcosis using an independent cohort of 22 patients with alveolar echinococcosis and 22 healthy individuals. While hsa-miR-125b-5p may serve as a promising diagnostic marker for alveolar echinococcosis, additional experiments should be performed to further investigate its diagnostic value. Future studies are required to identify the expression of hsa-miR-125b-5p in the plasma of patients with cystic echinococcosis, alveolar echinococcosis, and other liver diseases to determine the potential of hsamiR-125-5p as a differential biomarker.

- Declarations

\section{Declarations}

\section{Ethics approval and consent to participate}

This study was approved by the ethics review broad of the Hospital Affiliated of Qinghai University (approval number: P-SL-2019054).

\section{Availability of data and materials}

Not applicable.

\section{competing interests}

The author(s) declare that they have no competing interests.

\section{Consent for publication}


Agree to publish

\section{Authors' contributions}

CDP conceived and designed the study and drafted the manuscript. JBF did the research for q-PCR and data analysis. ZYG and PMQ helped design the study and revise the draft manuscript. All authors read and approved the final manuscript.

\section{Funding}

This work was supported by the Qinghai Science and Technology Department (grant numbers 2016-SFA5 and 2019-SF-131).

\section{Acknowledgements}

We would like to thank Editage (www.editage.cn) for English language editing.

\section{References}

1. Eckert J, Deplazes P. Biological, epidemiological, and clinical aspects of echinococcosis, a zoonosis of increasing concern. Clin Microbiol Rev. 2004;17:107-35.

2. Wang $\mathrm{H}$, Zhang JX, Schantz PM, et al. Epidemiologic survey and analysis on echinococcosis in humans and animals from 1995 to 2005 in Qinghai province. Zhongguo renshou gonghuanbing xuebao. 2006;22:1129-34. (in Chinese).

3. Nunnari G, Pinzone MR, Gruttadauria S, Celesia BM, Madeddu G, Malaguarnera G, Pavone P, Cappellani A, Cacopardo B. Hepatic echinococcosis: clinical and therapeutic aspects. World J Gastroenterol. 2012;18:1448-58.

4. Mariconti M, Bazzocchi C, Tamarozzi F, Meroni V, Genco F, Maserati R, Brunetti E. Immunoblotting with human native antigen shows stage-related sensitivity in the serodiagnosis of hepatic cystic echinococcosis. Am J Trop Med Hyg. 2014;90:75-9.

5. Gottstein B, Wang JH, Blagosklonov O, Grenouillet F, Millon L, Vuitton DA, Müller N. Echinococcus metacestode: in search of viability markers. Parasite. 2014;21:63.

6. Giraudoux P, Pleydell D, Raoul F, Quéré JP, Wang Q, Yang Y, Vuitton DA, Qiu J, Yang W, Craig PS. Transmission ecology of Echinococcus multilocularis: what are the ranges of parasite stability among various host communities in China. Parasitol Int. 2006;55:237-46.

7. Brunetti E, Kern P, Vuitton DA. Expert consensus for the diagnosis and treatment of cystic and alveolar echinococcosis in humans. Acta Trop. 2010;114:1-16.

8. Wang TP, Cao ZG,. Progress in prevention and control of Chinese hydatid disease and its existing problems. Zhongguo jishengchongxue yu jishengchongbing fangzhi zazhi. 2018;36(3):291-6. (In Chinese). 
9. Guo X, Zheng Y. Expression profiling of circulating miRNAs in mouse serum in response to Echinococcus multilocularis infection. Parasitology. 2017;144:1079-87.

10. Bartel DP. MicroRNAs: genomics, biogenesis, mechanism, and function. Cell. 2004;116:281-97.

11. Bartel DP. Micro RNAs: target recognition and regulatory functions. Cell. 2009;136:215-33.

12. Tao $\mathrm{YH}$, Wang $\mathrm{ML}$, Wang $\mathrm{M}$, et al. Quantification of circulating miR-125b-5p predicts survival in chronic hepatitis B patients with acute-on-chronic liver failure. Digestive Liver Disease. 2019;51:4128.

13. Wang J, Yan F, Zhao Q, Zhan F, Wang R, Wang L, Zhang Y, Huang X. Circulating exosomal miR-125a$3 p$ as a novel biomarker for early-stage colon cancer. Sci Rep. 2017;7:4150.

14. Jin X, Guo X, Zhu D, Ayaz M, Zheng Y. miRNA profiling in the mice in response to Echinococcus multilocularis infection. Acta Tropica. 2017;166:39-44.

15. Lin ZK, Zhou K, Lu J, et al. ROC curve analysis of mi RNA-RNA-221/222 as a marker for invasive papillary thyroid carcinoma in clinical value. Aizheng jinzhan. 2018;16(1):49-52. (In Chinese).

16. Mogahed NF, Khedr SI, Ghazala RA, Masoud IM. Can miRNA712-3p be a promising biomarker for early diagnosis of toxoplasmosis? Asian Pac. J Trop Med. 2018;11:688-92.

17. Liu J, Cao DP, Zhang XF, et al. Analysis of serum miRNAs expression spectrum in patients with alveolar echinococcosis. Chinese high altitude medicine biology. 2019;40:51-6. (in Chinese).

18. Jiang BF, Sha CQ, Zhang YG, et al. Expression of hsa-miR-125b-5p in serum of patients with alveolar echinococcosis and screening of its signal transduction pathway and target genes. Chinese high altitude medicine biology. 2020;41:39-45. (in Chinese).

19. Lee YS, Kim HK, Chung S, Kim KS, Dutta A. Depletion of human micro-RNA miR-125b reveals that it is critical for the proliferation of differentiated cells but not for the down-regulation of putative targets during differentiation. J Biol Chem. 2005;280:16635-41.

20. Morelli E, Leone E, Gallo, Cantafio,et al. Selective targeting of IRF4 by synthetic microRNA-125b-5p mimics induces anti-multiple myeloma activity in vitro and in vivo. Leukemia. 2015;29:2173-83.

\section{Figures}




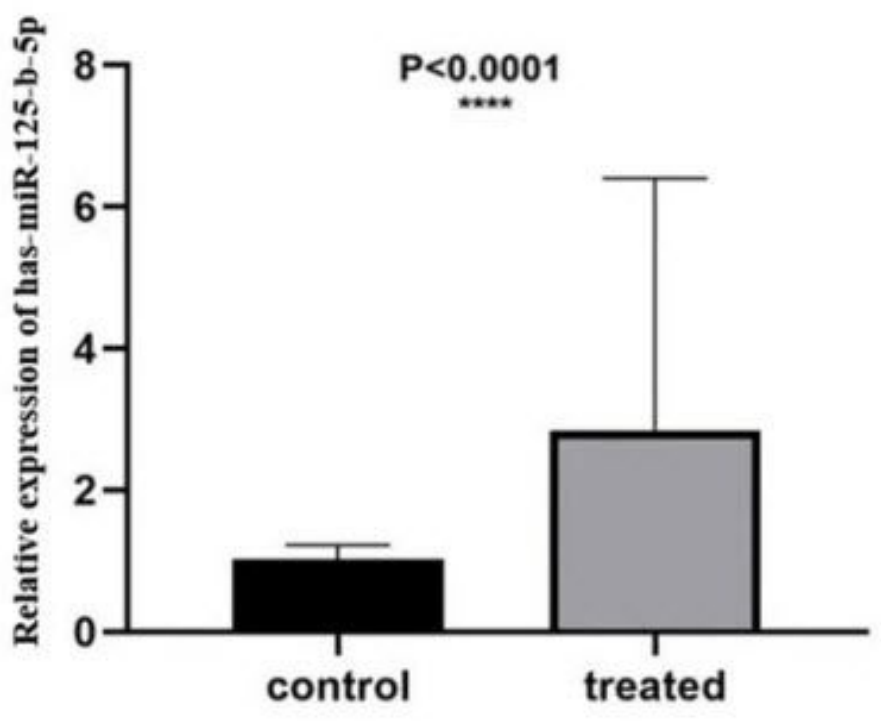

ROC curve: ROC of hsa-miR-125b-5p

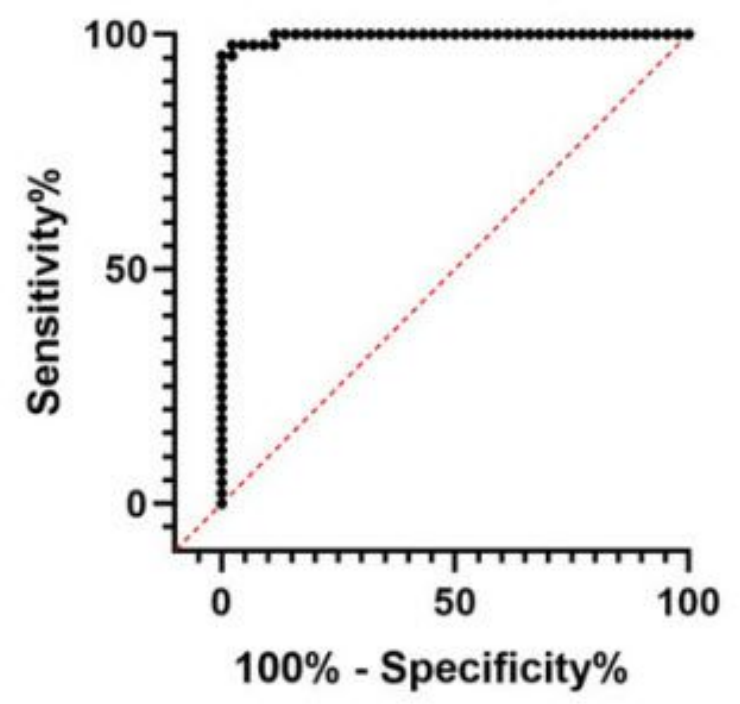

Figure 1

Relative expression of hsa-miR-125b-5p in the plasma of healthy (control) and AE (treated) patients (left). The receiver operating characteristic curve for the relative expression of hsa-miR-125b-5p in plasma in the controls and AE patients. AE, alveolar echinococcosis.
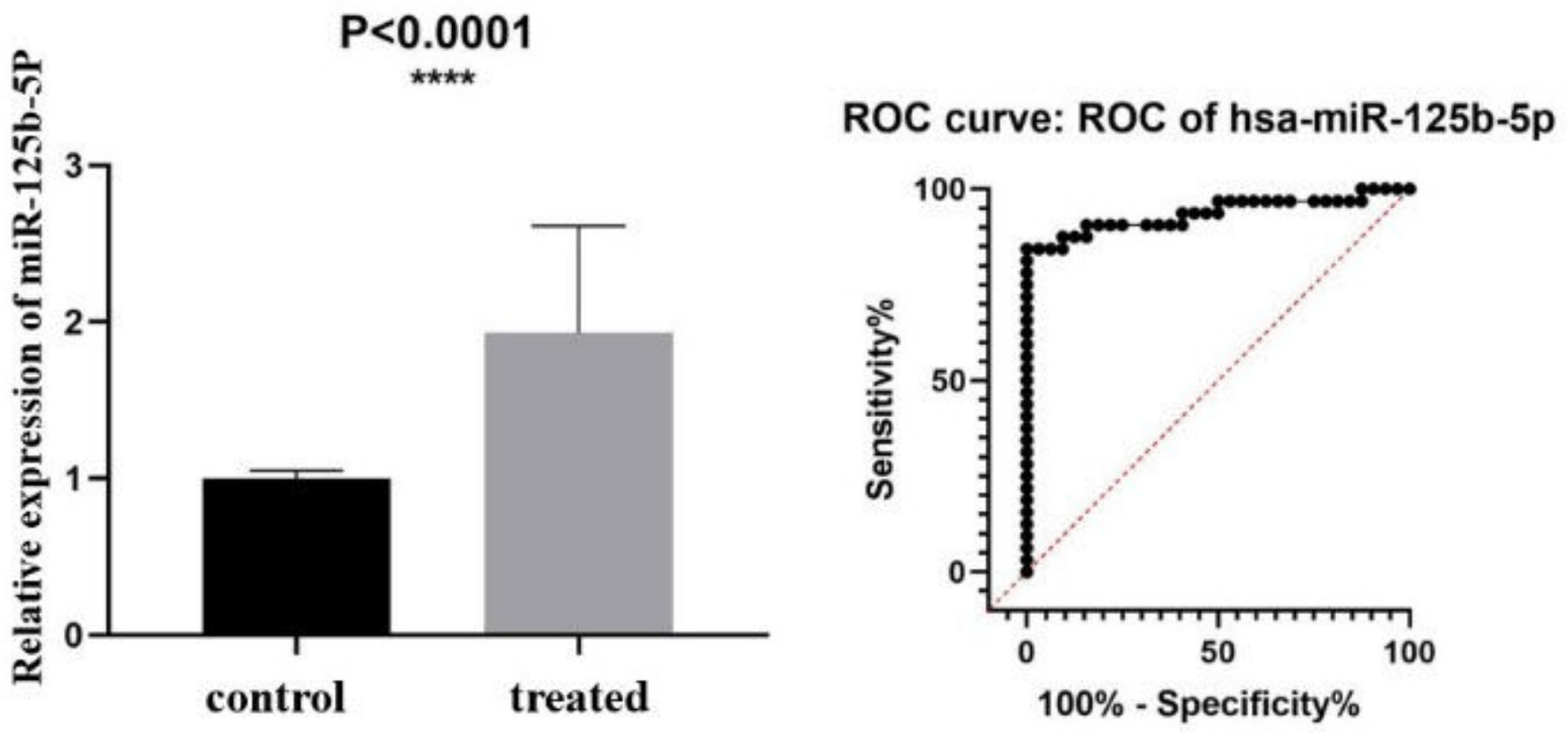

Figure 2

Relative expression of hsa-miR-125b-5p in AE and normal liver tissues. The receiver operating characteristic curve for the relative expression of hsa-miR-125b-5p in $A E$ and normal liver tissues. $A E$, alveolar echinococcosis. 


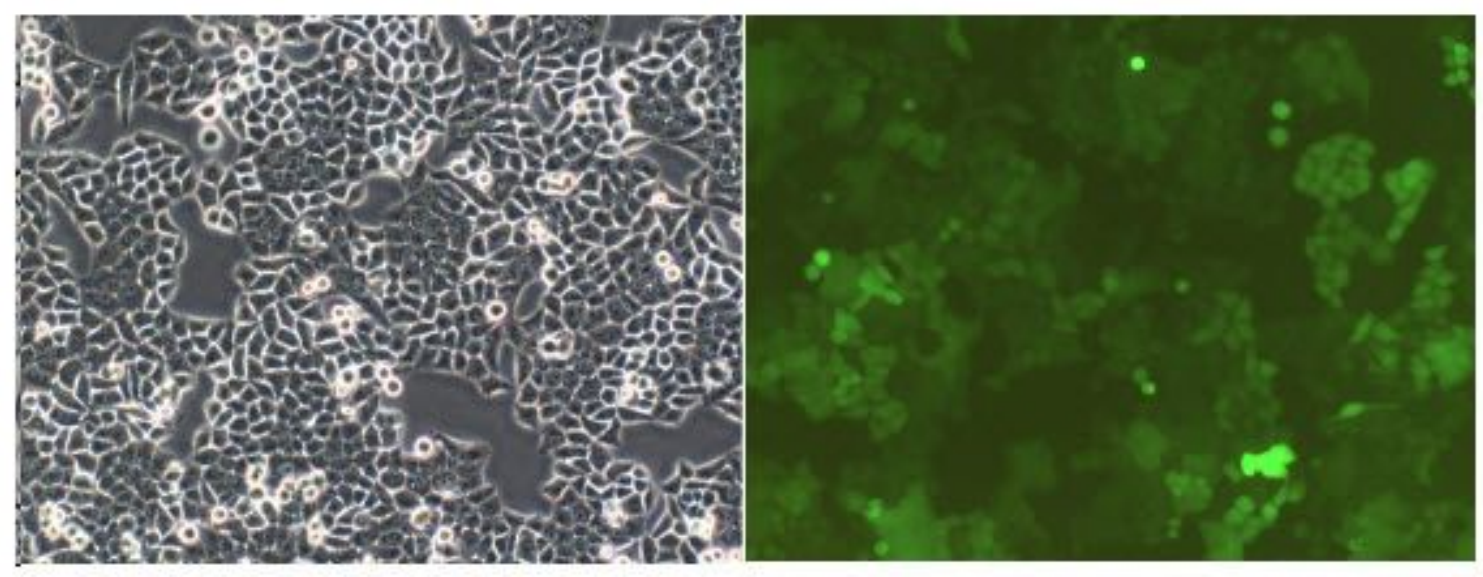

Figure 3

LV-hsa-miR-125b-5p inhibitor-transfected L-02 hepatocytes (magnification, 100x).

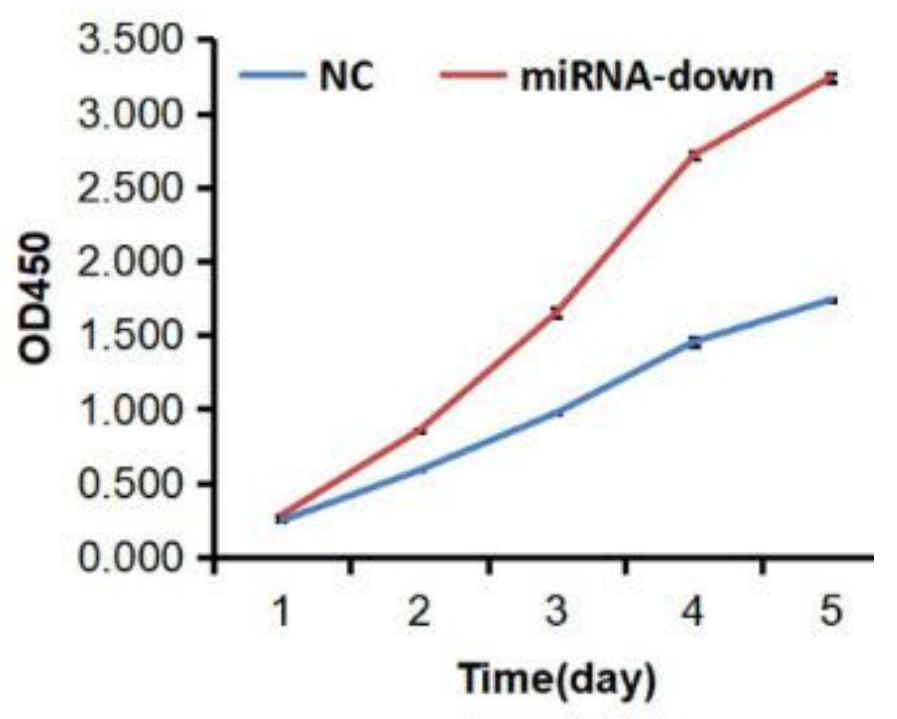

Figure 4

t-test analysis of the cell proliferation of LV-hsa-miR-125b-5p inhibitor-transfected and control cells (NC; P $<0.05)$. 


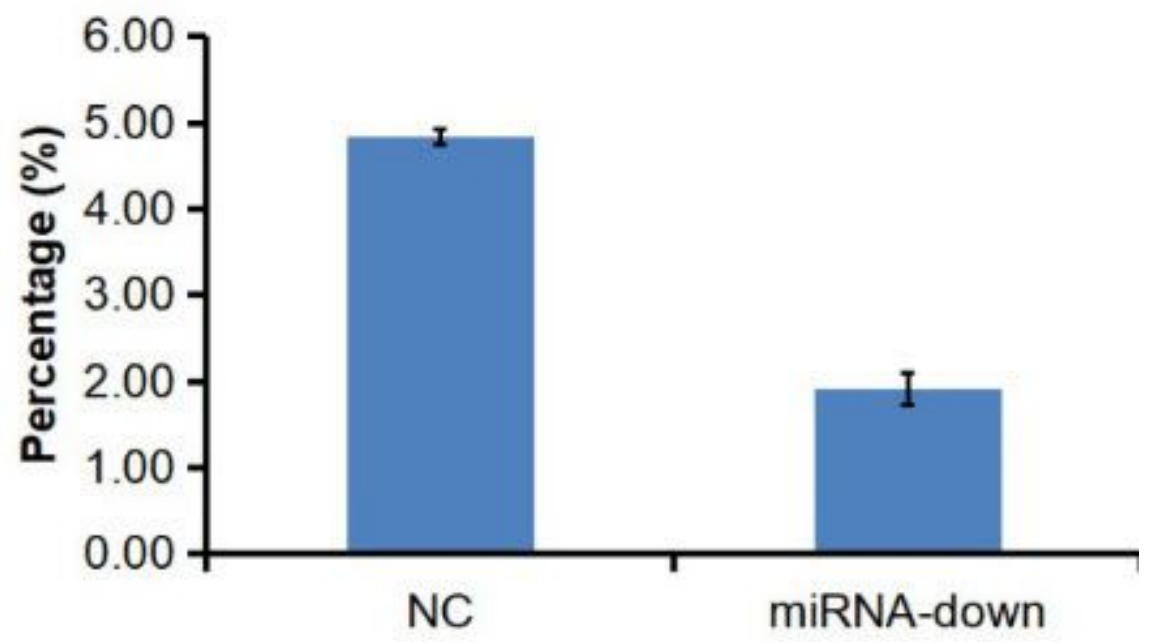

Figure 5

t-test analysis of apoptosis in control and LV-hsa-miR-125b-5p inhibitor-transfected L-02 cells $(P<0.05)$.

\section{Supplementary Files}

This is a list of supplementary files associated with this preprint. Click to download.

- ethicsreviewletter.jpg 